\title{
COUPLING CORRECTION AND BEAM DYNAMICS AT ULTRALOW VERTICAL EMITTANCE IN THE ALS*
}

\author{
C. Steier ${ }^{\dagger}$, D. Robin, A. Wolski, LBNL, Berkeley, CA94720, USA \\ G. Portmann, J. Safranek, SLAC, Menlo Park, CA94025, USA
}

\begin{abstract}
For synchrotron light sources and for damping rings of linear colliders it is important to be able to minimze the vertical emittance and to correct the spurious vertical dispersion. This allows one to maximize the brightness and/or the luminosity. A commonly used tool to measure the skew error distribution is the analysis of orbit response matrices using codes like LOCO. Using the new Matlab version of LOCO and 18 newly installed power supplies for individual skew quadrupoles at the ALS the emittance ratio could be reduced below $0.1 \%$ at $1.9 \mathrm{GeV}$ yielding a vertical emittance of about $5 \mathrm{pm}$. At those very low emittances, additional effects like intra beam scattering become more important, potentially limiting the minimum emittance for machine like the damping rings of linear colliders.
\end{abstract}

\section{INTRODUCTION}

The Advanced Light Source (ALS) is a third-generation source located at Lawrence Berkeley National Laboratory that has been operating for almost a decade and is generating forefront science over a broad area. However, the ALS was one of the first third-generation machines to be designed, and its performance will be outstripped by newer, more advanced machines. Accelerator and insertion device technology have changed significantly since the conception of the ALS, and in order to remain competitive in the core areas of high-resolution spectroscopy, high-spatialresolution soft x-ray microscopy, and experiments that exploit coherence, an upgrade plan is in place to enhance the performance.

The main possibilities to increase the brightness of the ALS are increasing the time-averaged current, reducing the beam size, and reducing the insertion device gaps. Currently those changes would result in (unacceptably) short lifetime. With continuous injection (top-off), the importance of this lifetime impediment will become significantly reduced in the future. Fig. 1 shows a comparison of the brightness of planned, new ALS insertion devices with the upgraded beam parameters to the typical brightness of a current ALS undulator. One of the main improvements comes from a smaller vertical emittance and the smaller physical gaps of the undulators.

\footnotetext{
* This work was supported by the U.S. Department of Energy, under Contract No. DE-AC03-76SF00098 and DE-AC03-76SF00515.

†CSteier@1bl.gov
}

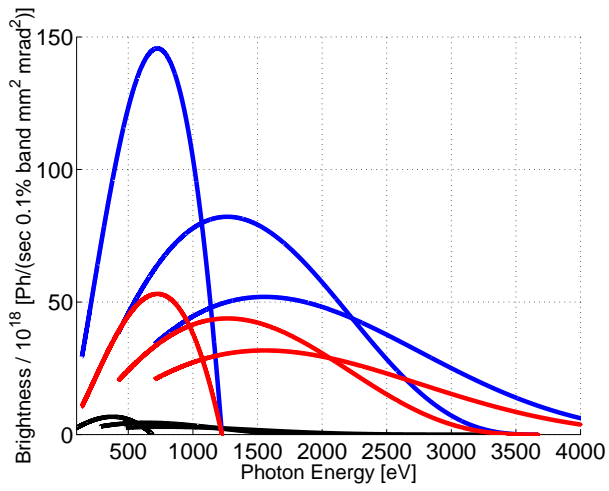

Figure 1: Comparison of brightness of new ALS insertion devices with $750 \mathrm{~mA}$ and $10 \mathrm{pm}$ vertical emittance (red/blue) to the typical brightness of a current U5 undulator (black).

\section{SIMULATION OF EMITTANCE CORRECTION}

A number of simulations was performed to find the smallest number of individual skew quadrupoles which still allows an effective emittance correction, as well as a good distribution of those skew quadrupoles and finally an optimum correction algorithm. The result was that 12 skew quadrupoles (one in each sector) were sufficient, six of them located at high dispersion points and six of them at lower dispersion points with a larger product of horizontal and vertical beta-functions. In the simulations many different minimization algorithms were used, but it turned out, that orbit response matrix analysis (using Matlab LOCO [1, 2]) to fit an effective skew quadrupole distribution gave results as good as the best other minimzation algorithm. Since it has the advantage of requiring very few iterations and automatially providing a measurement of the local coupling everywhere along the ring it is perfectly suited to be used with measurement data on the real machine. The simulations were also used to optimize the parameters of LOCO like the weight factor of the dispersion function, the outlier rejection tolerance, and the number of singular values. Fig. 2 shows the results of one of those simulation runs for 100 random seeds of misaligned machines. In this particular case it was possible to correct the vertical emittance below $5 \mathrm{pm}$ for $25 \%$ of the error seeds.

The skew quadrupoles at the ALS are integrated in the sextupoles and used to be connected in 4 chains. Based on the simulations, individual power supplies were installed last year, powering 18 individual skew quadrupoles - at least one in every sector. Two sectors have four skew quadrupoles each to generate a closed dispersion bump for 

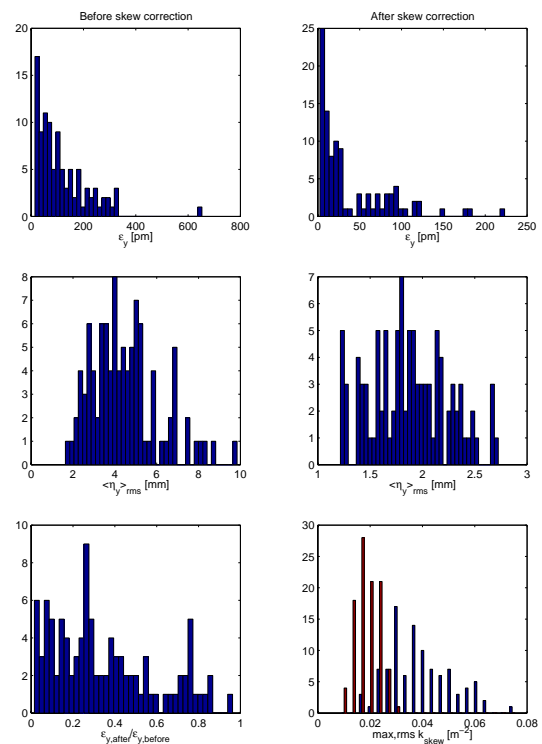

Figure 2: Histograms showing the results of simulated vertical emittance and dispersion correction for 100 randomly misaligned error seeds.

the fs-slicing experiments [3].

\section{MEASUREMENTS}

Because of the resolution limit even of x-ray beamlines it is quite difficult to measure emittances in the few pm range accurately. The primary beamline to measure emittances at the ALS uses an imaging optics with a KB-mirror pair, carbon filters to select the x-ray wavelength $(1.5 \mathrm{keV})$ and to attenuate, a BGO crystal to convert to visible light and a microscope with CCD. Even though the fundamental resolution limit would allow a direct measurement of emittances of a few pm, aberrations of the optics create a larger limit. Therefore we used three somewhat indirect but independent methods to measure the very small vertical emittances.

The first method was to determine the resolution of the beamline using Touschek lifetime measurements for various beamsizes. We then corrected the beamsize measurements for the beamline resolution to deduct the real vertical emittance. The second method was based on the analysis of an orbit response matrix, using a large number of skew gradient error fit parameters. With the calibrated machine model one can then calculate the vertical emittance using a lattice code. The final method used a scan of the RFacceptance while measuring the Touschek lifetime. For low RF amplitudes, the Touschek lifetime is strictly proportional to the bunch volume. Therefore one can deduct a very small emittance from a beamsize measurement at moderate coupling.

All three methods gave consistent results. In the best case, the measured vertical emittance as determined by the three methods was 4-7 pm, corresponding to an emittance ratio of less than $0.1 \%$ at $1.9 \mathrm{GeV}$ (natural emittance is $6.75 \mathrm{~nm}$ ). Fig. 3 shows an example of the change in beam- size and local tilt angle in one iteration of the emittance correction, The mean value of the emittance measurements of about $5 \mathrm{pm}$ is to our knowledge a world record for vertical emittances in electron/positron storage rings and is virtually identical to the design value for the NLC damping rings (references to vertical emittances achieved elsewhere are listed in [4]). It is interesting to note that emittances this small are within one order of magnitude of the theoretical limit due to the finite opening angle of the synchrotron radiation emission.
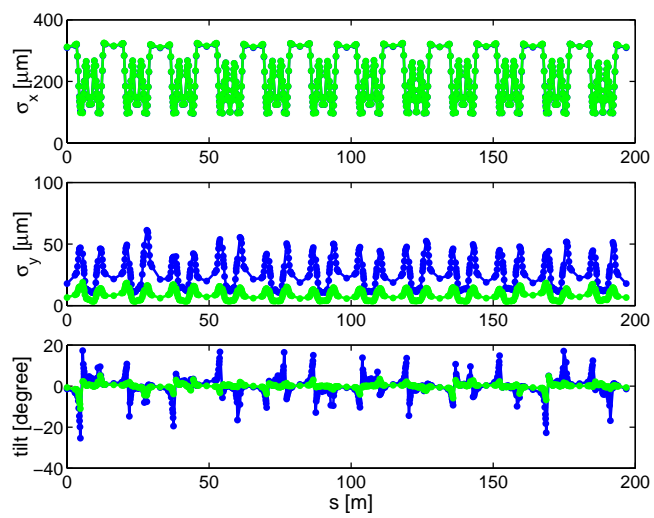

Figure 3: Horizontal/vertical beamsize and beam tilt as calculated from calibrated machine model using orbit resposne matrix analysis. The blue case is before coupling correction, the green one after one iteration.

\section{EMITTANCE CONTROL}

Until the full energy injector upgrade at the ALS is finished to allow top-off injection, it is important to still operate with artificially increased vertical emittance to achieve reasonable beam lifetimes for users. Historically at the ALS a controlled excitation of the nearby linear coupling resonance has been used to increase the vertical beamsize. After installing the individual skew quadrupoles, we switched to a different scheme. The emittance and vertical dispersion is corrected and then 12 skew quadrupoles are used such as to introduce a global vertical dispersion wave, without introducing coupling. In that case, the vertical emittance is generated directly via quantum excitation. The local emittance ratio around the ring can be made fairly flat and all local tilt angles are small.

The main advantages of this method are a better beamsize stability (especially for scanning undulators/wigglers), better dynamic (momentum) aperture and less sensitivity of the momentum aperture to the vertical physical aperture. An example of the improvement in longtime beamsize stability can be seen in Fig. 4.

\section{BEAM DYNAMICS AT LOW EMITTANCE}

\section{Single Particle Dynamics}

Since the lifetime at all (low energy) third generation light sources is (strongly) Touschek limited, the momentum 

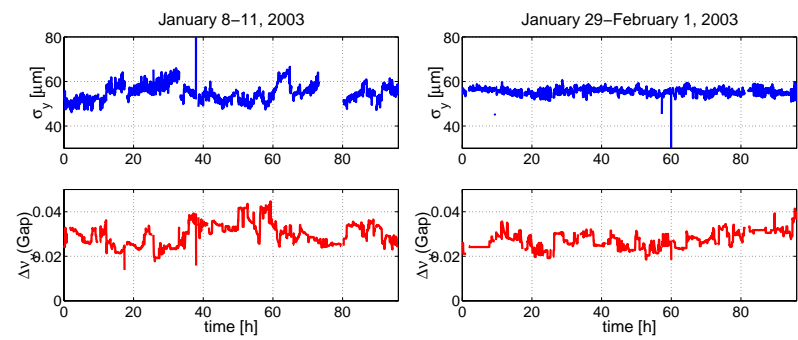

Figure 4: Vertical beamsize for two four day periods. Using a global vertical dispersion wave to increase the vertical emittance (right) leads to a much better beamsize stability than excitation of the coupling resonance (left).

aperture of the ring is very important [5]. For top-off operation, injection efficiency becomes very important as well (i.e. on-energy dynamic aperture). For most light sources the dominant factor for the momentum aperture is the transverse single particle dynamics and in most cases the particles are lost on the narrowest vertical apertures. Therefore it is important to understand and optimize the dependence of the momentum aperture on the vertical physical aperture.

We found that for the current ALS lattice the dependence of the momentum aperture (lifetime) on the vertical physical aperture became much weaker both for the case with corrected vertical emittance and the case with vertical dispersion wave, compared to the case with artificially excited coupling resonance (compare Fig. 5). Combined with the improvements in the lattice implemented two years ago and better correction of lattice symmetry errors this will enable the ALS to reduce the physical gap of insertion devices from the current $8-9 \mathrm{~mm}$ down to about $5 \mathrm{~mm}$.

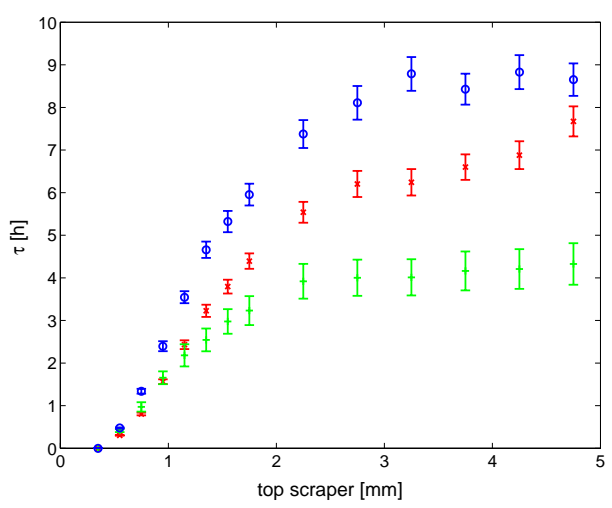

Figure 5: Measured lifetime of the ALS versus half aperture in one straight for three different cases: excited coupling resonance (red), corrected coupling and vertical dispersion (green), vertical dispersion wave (blue). The two cases with excited coupling resonance and dispersion wave were measured for identical vertical emittance.

Simulating the effects in tracking, we found good agreement between measurements and simulations. The simulations showed that a correction of the coupling reduces the sensistivity of the dynamic momentum aperture to the vertical physical aperture [6]. The injection efficiency for smaller vertical gaps was studied as well and no show stoppers were found so far. It is planned to install a collimator system to protect all insertion devices in the ALS from injection losses during top-off operation.

\section{Intra beam scattering}

Although it is not an important effect for the ALS, intra beam scattering is important for the damping ring designs for linear colliders or future light sources with smaller natural emittance than the ALS. To verify the accuracy of intra beam scattering calculations, a measurement program was started a few years ago mostly at the ATF [7] and also at the ALS [8]. At the ALS at that time only chains of skew quadrupoles were available, limiting the minimum vertical emittance at which the measurements could be performed. With an emittance ratio of about $0.75 \%$ at $1.5 \mathrm{GeV}$, no effects of IBS could be seen. At lower energies, significant effects of IBS were found, but since the minimum vertical emittances were relatively large, the quantitative separation of IBS effects and potential well distortion/microwave instability effects was difficult.

Since the installation of the individual skew quadrupoles it is now possible to achieve much smaller vertical emittances, so it should be possible to measure IBS effects in a much cleaner way. Therefore the IBS measurement program at the ALS has been restarted.

\section{SUMMARY}

It is planned to upgrade the performance of the ALS significantly over the coming years by upgrading the injector for full energy (top-off) injection, upgrading the rf-system to allow for higher current operation and installing new insertion devices (in-vacuum and superconducting). Studies to correct and control the vertical emittance were carried out with the goal to improve the brightness once the full energy injector is installed. Vertical emittances as small as $5 \mathrm{pm}$ were achieved which to our knowledge is currently the best value achieved worldwide. In addition studies were carried out which lead to big improvements in the vertical beamsize stability and dependence of the momentum aperture on the physical vertical aperture.

\section{REFERENCES}

[1] J. Safranek et al., Proceedings of EPAC2002, Paris (2002).

[2] A. Terebilo, et al., Proceedings of PAC2001, Chicago p. 3203 (2001).

[3] C. Steier et al., Accelerator Physics Challenges of the fsSlicing Upgrade at the ALS, these proceedings

[4] K. Kubo et al., Phys. Rev. Lett. 88:194801 (2002).

[5] C. Steier et al., Phys. Rev. E 65, 056506 (2002).

[6] D. Robin et al., Impact of Narrow Gap Chambers on the Lifetime at the ALS, these proceedings.

[7] K. Bane et al., Phys. Rev. STAB 5, 084403 (2002).

[8] C. Steier et al., Proceedings of PAC2001, Chicago p. 2938 (2001). 